\title{
Rhamnolipid Biosurfactants-Possible Natural Anticancer Agents and Autophagy Inhibitors
}

\author{
Severina Semkova ${ }^{1}$, Georgi Antov ${ }^{1,2}$, Ivan Iliev ${ }^{1,3}$, Iana Tsoneva ${ }^{1}$, Pavel Lefterov ${ }^{4}$, Nelly Christova ${ }^{5}$, \\ Lilyana Nacheva ${ }^{5}$, Ivanka Stoineva ${ }^{6}$, Lyudmila Kabaivanova ${ }^{5, *}$, Galya Staneva ${ }^{1}$ and Biliana Nikolova ${ }^{1, *(\mathbb{C})}$ \\ 1 Institute of Biophysics and Biomedical Engineering, Bulgarian Academy of Sciences, "Acad. G. Bonchev" Str., \\ Bl. 21, 1113 Sofia, Bulgaria; severina.yordanova@gmail.com (S.S.); antov8107@abv.bg (G.A.); \\ taparsky@abv.bg (I.I.); itsoneva@bio21.bas.bg (I.T.); gstaneva@bio21.bas.bg (G.S.) \\ 2 Institute of Plant Physiology and Genetics Bulgarian Academy of Sciences, "Acad. G. Bonchev" Str., Bl. 21, \\ 1113 Sofia, Bulgaria \\ 3 Institute of Experimental Morphology, Pathology and Anthropology with Museum, \\ Bulgarian Academy of Sciences, “Acad, G. Bonchev" Str., Bl. 25, 1113 Sofia, Bulgaria \\ 4 Faculty of Educational Studies and the Arts University of Sofia, Bul. Shipchenski Prohod, 1113 Sofia, Bulgaria; \\ lefterovpavel@gmail.com \\ 5 The Stephan Angeloff Institute of Microbiology, Bulgarian Academy of Sciences, "Acad. G. Bonchev" Str., \\ Bl. 26, 1113 Sofia, Bulgaria; nchrist@abv.bg (N.C.); lin1@abv.bg (L.N.) \\ 6 Institute of Organic Chemistry with Centre of Phytochemistry, Bulgarian Academy of Sciences, \\ "Acad. G. Bonchev" Str., Bl. 9, 1113 Sofia, Bulgaria; istoineva@yahoo.com \\ * Correspondence: lkabaivanova@yahoo.com (L.K.); nikolova@bio21.bas.bg (B.N.)
}

\section{check for} updates

Citation: Semkova, S.; Antov, G.; Iliev, I.; Tsoneva, I.; Lefterov, P.; Christova, N.; Nacheva, L.; Stoineva, I.; Kabaivanova, L.; Staneva, G.; et al. Rhamnolipid BiosurfactantsPossible Natural Anticancer Agents and Autophagy Inhibitors. Separations 2021, 8, 92. https:// doi.org/10.3390/separations 8070092

Academic Editors: Giorgio Marrubini and Caroline West

Received: 12 April 2021

Accepted: 23 June 2021

Published: 28 June 2021

Publisher's Note: MDPI stays neutral with regard to jurisdictional claims in published maps and institutional affiliations.

Copyright: (c) 2021 by the authors. Licensee MDPI, Basel, Switzerland. This article is an open access article distributed under the terms and conditions of the Creative Commons Attribution (CC BY) license (https:// creativecommons.org/licenses/by/ $4.0 /)$.

\begin{abstract}
Background/Aim: A number of biologically active substances were proved as an alternative to conventional anticancer medicines. The aim of the study is in vitro investigation of the anticancer activity of mono- and di-Rhamnolipids (RL-1 and RL-2) against human breast cancer. Additionally, the combination with Cisplatin was analyzed. Materials and Methods: Breast cell lines (MCF-10A, MCF-7 and MDA-MB-231) were treated with RLs and in combination with Cisplatin. The viability was analyzed using MTT assay, and investigation of autophagy was performed via acridine orange staining. Results: In contrast to the healthy cells, both tested cancer lines exhibited sensitivity to RLs treatment. This effect was accompanied by an influence on the autophagy-related acidic formation process. Only for the triple-negative breast cancer cell line (MDA-MB-231) the synergistic effect of the combined treatment $(10 \mu \mathrm{M}$ Cisplatin and $1 \mu \mathrm{g} / \mathrm{mL} \mathrm{RL}-2)$ was observed. Conclusion: Based on studies on the reorganization of membrane models in the presence of RL and the data about a higher amount of lipid rafts in cancer cell membranes than in non-tumorigenic, we suggest a possible mechanism of membrane remodelling by formation of endosomes. Shortly, in order to have a synergistic effect, it is necessary to have Cisplatin andRL-2 as RL2 is a molecule inducingpositive membrane curvature.
\end{abstract}

Keywords: bacterial natural rhamnolipids; breast cancer; autophagy; cytotoxity; membrane reorganization; endocytosis

\section{Introduction}

Recently, a great number of products with natural origin have been the subject of intensive research. Microbial surfactants have some advantages over their chemical counterparts: effectiveness in extreme $\mathrm{pH}$ and temperatures, less toxicity, etc. [1]. Among other biosurfactants, Rhamnolipids (RLs) present a group of chemical structures with amphiphilic character. They are composed of $\beta$-hydroxy fatty acid connected by the carboxyl end to a rhamnose sugar molecule. Rhamnolipids are predominantly produced by Pseudomonas aeruginosa and classified as mono- and di-Rhamnolipids [2]. Rhamnolipids draw attention to their prominent cytotoxic effects, on diverse cancer cell lines, due to well-expressed surface activity. As secondary metabolites, they play essential roles in 
the survival of their producing microorganisms by facilitating nutrient transport, interfering in microbe-host interactions and quorum sensing mechanisms, or by acting as biocide agents [3]. The novel structures of Rhamnolipids reveal diverse biological activities. They exhibit properties such as low toxicity, high biodegradability and are obtained from renewable sources. Due to these properties, RLs seem applicable in biomedicine [4].

In several studies, the anticancer properties of Rhamnolipids, isolated from different Pseudomonas aeruginosa strains, have been reported $[5,6]$. Thanomsub et al. have identified two Rhamnolipids: (Rha-Rha $\mathrm{C}_{10}-\mathrm{C}_{10}$ ) and (Rha-Rha $\mathrm{C}_{10}-\mathrm{C}_{12}$ ), both with similar surfactant activity but with different antiproliferative properties [7]. Similar results have been reported lately by Zhao et al. Rhamnolipids include two main types, mono- (RL-1) and di-Rhamnolipids (RL-2) [8]. Di-Rhamnolipids showed significant antiproliferative activity against the human breast cancer cell line (MCF-7). Relevant data have been acquired in our previous study where Rhamnolipid fraction 1 (RL-1) showed a significant antiproliferative effect against a panel of leukemic cells [5]. However, very little is known about the molecular basis of the action of this biosurfactant. The process of induced tumor cell death requires prolonged exposure, which suggests that multiple mechanisms leading to a cascade of events are involved in the destruction of leukemic and human cancer cells [5]. Sanchez et al. [9] have shown that di-Rhamnolipids are able to induce permeabilization in human erythrocytes by detergent-like mechanism leading to morphological changes due to partition of the outer plasma membrane and/or cytoskeleton reorganization. Recently, by using electroformed large unilamellar vesicles with lipid rafts, Come et al. [10] observed membrane budding after treatment with Rhamnolipids. The results $[9,10]$ are very interesting, but the subjects of their studies are the model membranes and human erythrocytes, which differ from somatic cells and even more from cancer cells.

The ability of biosurfactants, especially Rhamnolipids, is to control various cellular functions in mammalian cells, such as: growth inhibition, autophagy, apoptosis, necrosis, migration and cell cycle arrest, and are a function of their potential to act as anticancer agents [11].

Autophagy was described as a type of apoptosis, sharing some common regulatory elements, responsible for maintaining the intracellular metabolic homeostasis $[12,13]$. It is still unclear if autophagy leads to cell survival or cell death in the context of environmental stress factors such as metabolic deprivation or DNA damage caused by anticancer chemoor radiation therapy. [14,15].

It is interesting to follow whether natural bioproducts with surfactant properties can facilitate or decrease the autophagy process in normal and cancer cells. Furthermore, their possible contribution to the action of conventional anticancer drugs should be investigated.

The aim of the present study is to evaluate RLs' cytotoxic effect and its effect on autophagy toward two different breast cancer cell lines-low metastatic MCF-7, high metastatic MDA-MB-231 and a non-tumorigenic cell line as a control-MCF-10A. Moreover, the combination of Rhamnolipids and the conventional anticancer drug Cisplatin is investigated.

\section{Materials and Methods}

\subsection{Rhamnolipids-Microorganism, Media, Cultivation and Purification}

Pseudomonas aeruginosa BN10 strain was isolated from soil polluted with hydrocarbons by standard enrichment techniques as described previously [16]. The selection of isolates for surface activity was performed. Strain BN10 was maintained in nutrient agar slants (Difco Laboratories Inc., Detroit, MI, USA) at $4{ }^{\circ} \mathrm{C}$. Cultures were incubated while shaking $(120 \mathrm{rpm})$ at $30^{\circ} \mathrm{C}$ for 7 days. The resultant culture broth was centrifuged at $8000 \times g$ for $20 \mathrm{~min}$. The supernatant was acidified with $6 \mathrm{M} \mathrm{HCl}$ and allowed to stay at $4{ }^{\circ} \mathrm{C}$ overnight. After another centrifugation at $12,500 \times g$ for $20 \mathrm{~min}$, the precipitate was collected. The extraction was accomplished by equal volume of ethyl acetate. The organic phase was removed under reduced pressure at $40{ }^{\circ} \mathrm{C}$ and the resulting light-brown-colored viscous liquid was considered as the crude biosurfactant. 
The biosurfactant-containing extract was dissolved in $3 \mathrm{~mL}$ of chloroform/methanol/ water $(65: 15: 2 v / v / v)$ and afterward purified by using normal pressure chromatography on silica gel 60 (particle size 0.063-0.200 mm, mesh size 70-230; Merck, Darmstadt, Germany). The surface-active compounds were eluted with dichloromethane/methanol/water $\left(\mathrm{CH}_{2} \mathrm{C}_{12} / \mathrm{CH}_{3} \mathrm{OH} / \mathrm{H}_{2} \mathrm{O}\right) 65: 15: 2$, and fractions $(1.5 \mathrm{~mL})$ were collected. The purification of the Rhamnolipids was monitored offline by thin-layer chromatography (TLC). TLC was carried out on Si gel 60 plates as stationary phase with the same solvent system as used for normal pressure chromatography and spraying with orcinol/sulfuric acid reagent for detection of Rhamnolipid compounds. The purified fractions were compared with reference substances of Rhamnolipids from Jeneil, Biosurfactant Jeneil Biotech Inc. Saukville, WI, USA.

\subsection{Anticancer Drug}

The conventional anticancer drug, Cisplatin (CisPt) (purchased from Accord Health Care LTD Whiddon Valley Barnstaple, Devon, UK), was used in the study. Forty-eight hours after treatment (range 0-100 $\mu \mathrm{M}$ ), the cells were subject to different assays.

\subsection{Cell Lines and Culture Conditions}

Three human breast epithelial cell lines purchased from the American Type Culture Collection-ATCC (Manassas, VA, USA) were used to perform experiments. The nontumorigenic breast epithelial cell line (MCF-10A), used as control, was cultivated in Dulbecco's Modified Eagle's Medium (DMEM) (Sigma-Aldrich, St. Louis, MO, USA) supplemented with $10 \%$ fetal bovine serum (FBS), $1 \%$ sodium pyruvate, $1 \%$ MEM Non-Essential Amino Acids (NEAA), $20 \mathrm{ng} / \mathrm{mL}$ human epithelial growth factor (hEGF), $10 \mu \mathrm{g} / \mathrm{mL}$ insulin, $0.5 \mu \mathrm{g} / \mathrm{mL}$ hydrocortisone without antibiotics. The adenocarcinoma cell lines (MCF-7 and MDA-MB-231) were cultivated in DMEM, supplemented with $10 \%$ FBS, $1 \%$ sodium pyruvate and $1 \%$ NEAA without antibiotics. All cell lines were grown in an incubator at $37^{\circ} \mathrm{C}$ and a humidified atmosphere with $5 \% \mathrm{CO}_{2}$.

\subsection{Cytotoxicity Assessment}

The cytotoxic activity of Rhamnolipids on the cultivated cells was measured by MTT [3-(4,5-dimethylthiazol-2-yl)-2,5-diphenyl tetrazolium bromide] colorimetric test [17]. After overnight incubation, the cells were treated with different concentrations of Rhamnolipids alone or in combination with Cisplatin and incubated for additional $48 \mathrm{~h}$. Used concentrations for test compounds were: $0-10 \mu \mathrm{g} / \mathrm{mL}$ range for Rhamnolipids and $0-100 \mu \mathrm{M}$ for Cisplatin, respectively. For combined treatment, fixed $1 \mu \mathrm{g} / \mathrm{mL}$ Rhamnolipids (RL-1 or RL-2) concentration and all variations Cisplatin were used. Later, the MTT solution ( $20 \mu \mathrm{L}$ of $5 \mathrm{mg} / \mathrm{mL}$, Sigma-Aldrich, USA) was added to each well, and the plates were re-incubated for additional $4 \mathrm{~h}$. Finally, the medium was removed, and $100 \mu \mathrm{L}$ of lysis buffer $(10 \%$ SDS, $0.01 \mathrm{M} \mathrm{HCI})$ was added to solubilize the formed formazan crystals. The amount of formazan crystal was determined by measuring the absorbance at $570 \mathrm{~nm}$ using a Thermo Multiskan Spectrum spectrophotometer (Thermo Fisher Scientific, Waltham, MA, USA), and the half-maximal inhibitory concentration $\left(\mathrm{IC}_{50}\right)$ of the pure compound was calculated by Origin software. All experiments were performed in triplicate, at least three times independently.

\subsection{Acridine Orange (AO) Staining}

The cells were seeded at glass-bottom dishes (MatTek Life Science Corr. Ashland, MA, USA) with density of $2 \times 10^{4}$ cells / $\mathrm{mL}$ in duplicates and incubated for $24 \mathrm{~h}$ for full adhesion. After incubation, the breast cells were treated with previously calculated $\mathrm{IC}_{50}$ dosages of mono- and di-Rhamnolipids in fresh culture medium. As controls, untreated cells were used. Forty-eight hours after treatment, Acridine Orange staining was performed. Briefly, cells were stained with $1 \mu \mathrm{g} / \mathrm{mL}$ Acridine Orange $(2.7 \mu \mathrm{M})$ solution in complete culture medium for $15 \mathrm{~min}$ at room temperature. After washing, fluorescent images 
were acquired using Leica DMIL microscope equipped with $20 \times$ and $40 \times$ objectives and Moticam 2500 camera.

\subsection{AO Signal Intensity}

The AO intensity was calculated using a simple 5-step method. As the amount of light produced by biofluorescence is rather small and exposure duration was relatively short, no background noise was present in the original images; therefore, no denoising was needed. The first step was selecting cell line images treated with RL 1, RL 2 and untreated control of equal exposure time. In step 2 , each of the sample images was then split into separate 8-bit red, green and blue channels where minimum signal intensity is represented by 0 , and maximum signal intensity is represented by 255 . The blue channel was immediately discarded as it was irrelevant. In step 3, the value of each pixel in the green channel was subtracted from the value of the corresponding pixel in the red channel. In step 4 , the negative values produced by the previous step were set to the value of 0 . In step 5, the values of all pixels in the red channel were summed and then divided by the number of cells present in the image. This number was further divided by the magnification of the microscope. The results were obtained using the standard image and data processing libraries PIL and NumPy.

The following method was found to precisely filter out only the signal produced by acridine orange and represents the amount of orange light captured by the camera sensor per cell in the image.

\subsection{Flow Cytometry}

Flow cytometry analysis was performed by using Annexin V-FITC apoptosis detection kit (Sigma-Aldrich St. Louis, MO, USA). After $48 \mathrm{~h}$ RL incubation, the cells were trypsinized and labeled with the fluorochromes at room temperature, according to the manufacture's instructions. The samples were measured by flow cytometry (LSR II, BD Biosciences) and analyzed with BD FACS DIVA software (BD Biosciences).

\subsection{Evaluation of the Effect of Combined Treatment}

In order to predict the type of effect of combined treatment, we calculated the theoretical values of cell viability. For this purpose, the used the equation was:

$$
c=a \times b / 100
$$

where $\mathrm{a}$ and $\mathrm{b}$ are cell survival values in \% after treatment with Cisplatin and $1 \mu \mathrm{g} / \mathrm{mL}$ of RL-1 and RL-2, respectively. The theoretical values were calculated for each separated point and were compared with the real value after combined treatment. If $c_{\text {measured }}=c_{\text {calculated }}$ the combined effect is additive; if $\mathrm{c}_{\text {measured }} \leq \mathrm{c}_{\text {calculated }}$ the combined effect is considered to be synergetic; if $c_{\text {measured }} \geq c_{\text {calculated }}$ the effect is antagonistic [18].

\subsection{Statistical Analysis}

Statistical analysis was performed using Microsoft Excel and Origin software. All data are presented as means \pm SD For statistical significance evaluation of experimental data, a comparison between: treated and control probes; cancer and normal cell lines were performed by Student's t-test. Each $p$-value lower than 0.05 was considered statistically significant.

\section{Results and Discussion}

The current study is an attempt to investigate the possibilities for anticancer effectiveness of mono- and di-Rhamnolipids (RL-1 and RL-2) against human breast cancer cell lines. The results for cytotoxicity properties of used Rhamnolipids fractions, explored via MTT assay, are presented in Figure 1. The data are examined $48 \mathrm{~h}$ after the treatment, and the survival rate of cells was estimated as a percentage of untreated control. Based on data from Figure 1, we calculated the half-maximum inhibitory concentration $\left(\mathrm{IC}_{50}\right)$ values as follows: for RL-1 MCF-10-A $11.82 \mu \mathrm{g} / \mathrm{mL}$; MCF-7 $8.68 \mu \mathrm{g} / \mathrm{mL}$ and MDA-MB-231 
$6.99 \mu \mathrm{g} / \mathrm{mL}$; for RL-2 MCF-10-A $13.83 \mu \mathrm{g} / \mathrm{mL}$; MCF-7 $8.67 \mu \mathrm{g} / \mathrm{mL}$ and MDA-MB-231 $8.60 \mu \mathrm{g} / \mathrm{mL}$.
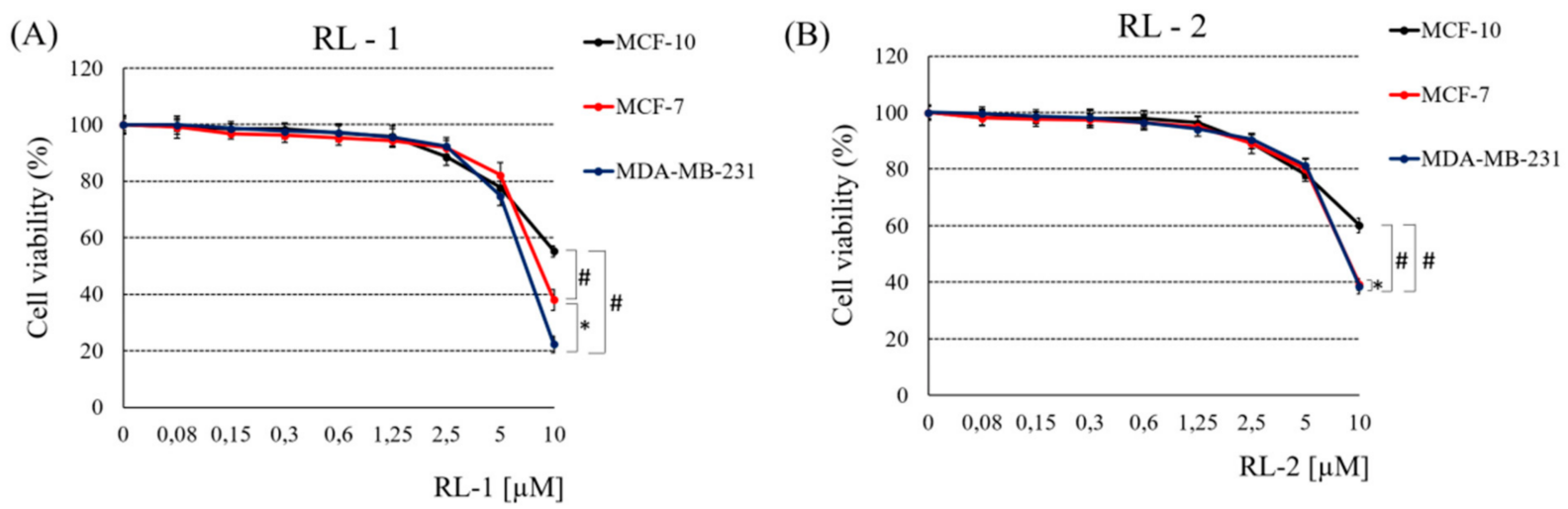

Figure 1. Drug-response curves based on measurement of cell viability of breast cell lines $48 \mathrm{~h}$ after treatment with RL-1 (A) and RL-2 (B), analyzed by MTT assay. All data are means \pm SD from three independent experiments (\# $p<0.05$ versus MCF-10A normal cells, ${ }^{*} p<0.05$ MCF-7 cell versus MDA-MB-231 cells; all other variations versus corresponding controls are statistically significant).

The results for MCF-10A cells showed slight influence on cellular viability for both applied Rhamnolipids compared to the demonstrated effect on cancer cell lines, which indicated less cytotoxicity of tested concentrations towards non-cancer cells, which appeared to be the best scenario for anticancer applications. Moreover, in contrast to the majority of anticancer agents, our data showed a stronger cytotoxic effect on high metastatic MDA-MB-231 cells (Figure 1A,B-blue lines) in comparison to the low metastatic cancer line MCF-7 (Figure 1A,B) - red lines) for both RL-1 and RL-2. The presented data indicated that mono-Rhamnolipids were more effective than di-Rhamnolipids. It had been long-discussed that mono-/di-Rhamnolipids affect healthy/cancer cells in opposite and different ways-some inhibited the growth of cancer cells, others of healthy ones $[7,19,20]$. Nevertheless, all authors concluded that a link existed between the nature of cancerous and normal cells and chemical composition, purity and level of surface tension reduction of applied Rhamnolipids and their effectiveness [6]. Based on our knowledge and the data obtained, we could suggest that the demonstrated difference in RL-1 and RL-2 action was due to stronger interaction with the cell surface, leading to a higher impact on cell survival, as well as differences in the metastatic potential of breast cancer lines.

In order to understand, even partially, the mechanism of action of the tested Rhamnolipids, the next part of the study was focused on the investigation of the possible connection with autophagy processes. We have assumed a link between autophagy and anticancer activity of biosurfactants because: (i) it is a well-known fact that glycolipids are membrane-acting molecules; (ii) a morphological change and generated vesicular membrane organelles after application of used Rhamnolipids (images not shown) were noticed in phase-contrast images (taken via microscopy). Autophagy plays a critical role in maintaining cellular homeostasis at both the cell-intrinsic and -extrinsic levels, adapting both normal and malignant cells to stress [21]. It has been reported that cancer cells could induce autophagy mechanisms against chemotherapeutic drugs, which helps cells to evade apoptotic pathways [22]. The development of the autophagy process is related to the generation of acidic vesicular organelles (AVOs) (such as lysosomes and autolysosomes), and one of the quickest, most reliable and accessible techniques for AVOs detection is Acridine Orange (AO) labeling [23]. AO is a commonly used cell-permeable fluorescent dye. When this probe enters and is localized into acidic compartments (AVOs), it emits orange and red fluorescence and could be easily imaged/measured via fluorescent microscopy [23].

Figure 2 shows fluorescent images taken after the AO labeling procedure on the studied breast cell lines are shown, performed $48 \mathrm{~h}$ after treatment with the calculated 
$\mathrm{IC}_{50}$ dosages of RL-1 and RL-2. All untreated (control) cells (from each cell line) stained with AO showed green fluorescence areas (including: cytoplasm, nucleus, nucleolus) and clear yellow/orange and red fluorescent dots, which indicates the presence of AVOs. These formations are expected, taking in mind that the formation of acidic vesicular structures is one of the main characteristics of cells engaged in autophagy, which as a recycling process plays an important role in maintaining normal cellular physiological condition [24,25]. Moreover, autophagy is considered to be one of the main mechanisms triggered in response to starvation, radiation, drugs action, etc. [24]. Thus, it should be expected that the number of AVOs will increase after treatment with Rhamnolipids if their cellular accumulation is related to the development and acceleration of autophagy. Surprisingly, AO staining images, obtained after treatment with RL-1 and RL-2, show different picture for cancer cell lines-the evident decrease in number and intensity of labeled AVOs. If we compare RL-treated healthy MCF-10A cells (Figure 2-upper panel) with cancer cell lines (Figure 2middle and lower panels), this observation is even clearer. The number and intensity of labeled AVOs in cancer lines increase in the order MCF-7 > MDA-MB-231.
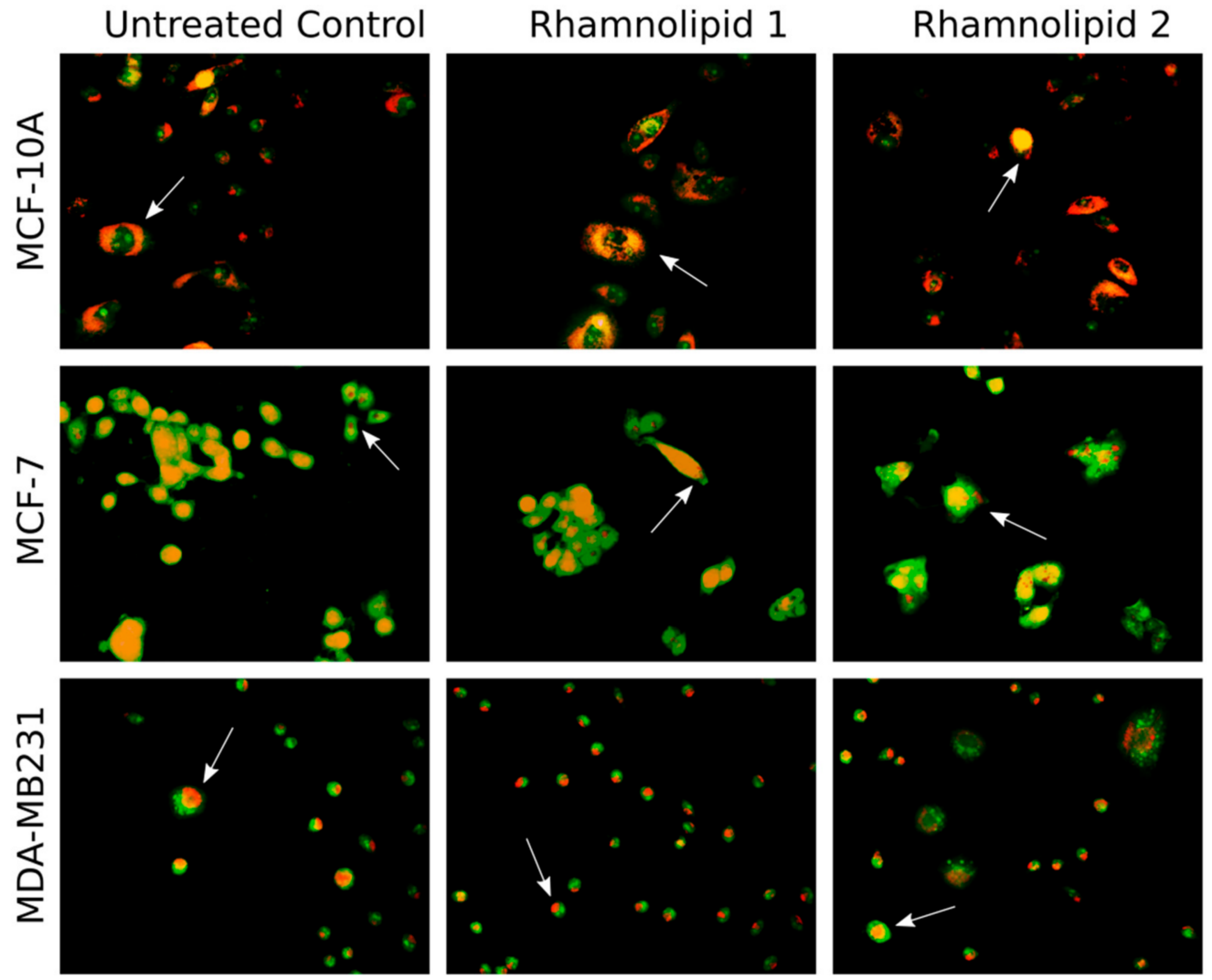

Figure 2. Acridine orange staining $48 \mathrm{~h}$ after treatment with $\mathrm{IC}_{50}$ dosages of RL-1 and RL-2 (MCF10A-upper panel, MCF-7-middle panel MDA-MB-231-lower panel). The arrows pointed to the AVOs. Magnification $20 \times$.

The digital data extracted from the images of Figure 2 are presented in Figure 3.

Hereby, we can conclude that after application of Rhamnolipids on the breast cancer cells, they demonstrate no autophagy-related acidic vesicular formation effects. We suggest that after treatment with RL-1 and RL-2, the possibilities for AVOs generation are violated, which might trigger pro-apoptotic signals, leading to apoptosis, respectively decrease in cancer cell lines viability. This assumption is in accordance with the acquired data from the MTT assay shown in Figure 1. Moreover, we could explain our results with the admission that Rhamnolipids have a destructive role for the already generated AVOs via induction of a process analogical to another well-known one: lysosomal membrane permeabilization 
(LMP). The connection between LMP (a membrane destabilization process) and triggering of cell death, based mainly on the uncontrolled release of cathepsins and other lysosomal enzymes, cytosolic acidification and necrosis, has been described in detail before [26], as well as an LMP-like a cancer cell killing mechanism [27].

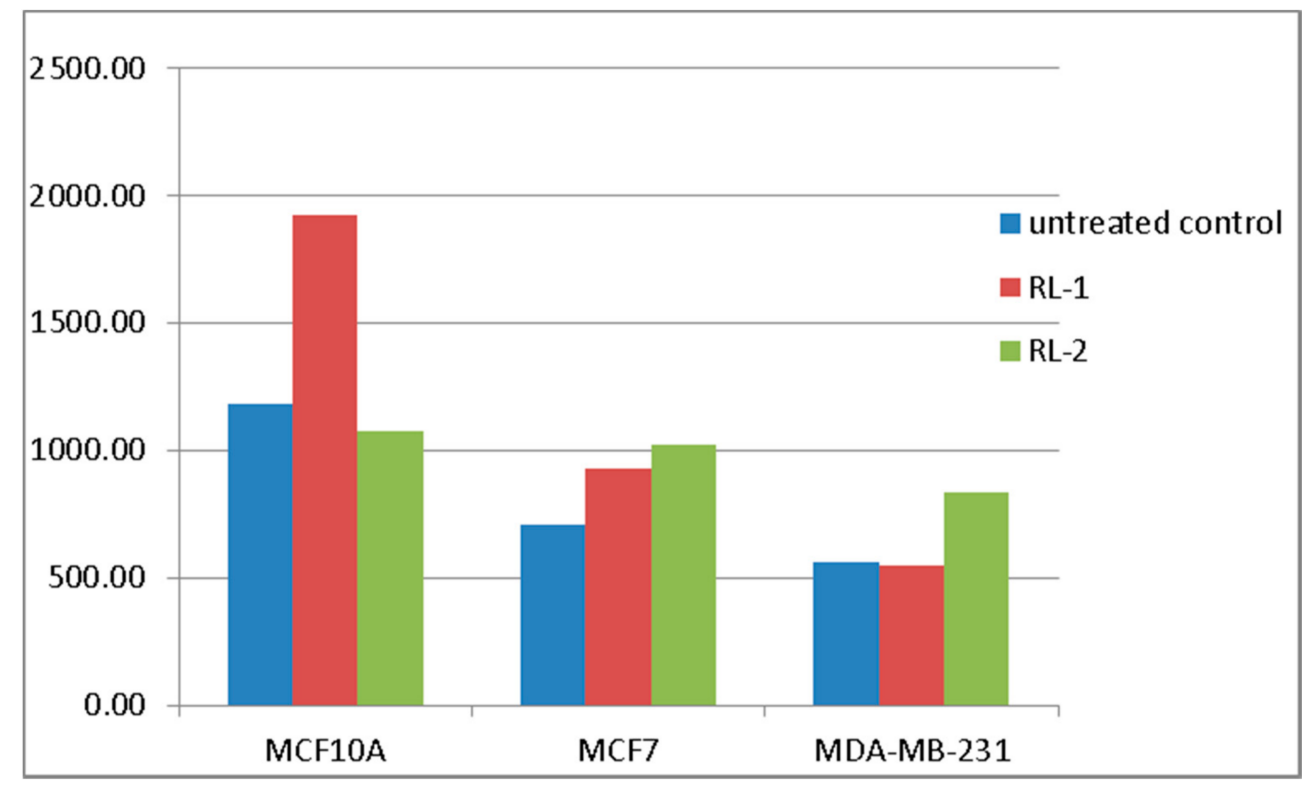

Figure 3. Acridine orange signal intensity after treatment with $\mathrm{IC}_{50} \mathrm{RL}-1$ and RL-2.

In the final part of the study, we tried to answer an additional question: what would happen with Rhamnolipid-induced cytotoxic activity if we combined them with another anticancer agent? However, if we assume that AVOs-formation decreased after the application of Rhamnolipids (probably via analogical LMP process), it is logical to expect that combination with another antineoplastic drug would possess an additive or synergistic effect and could additionally decrease cancer cell viability. Therefore, we performed MTT assay $48 \mathrm{~h}$ after combined treatment with Rhamnolipids and Cisplatin (data presented in Figure 4). In combined treatment (Figure 4), we used the maximum concentration of RL1 and RL2 at which no decrease in cell viability was observed compared to the negative control (there is no statistically significant difference between cell viability in the negative control and those treated with RL1 and RL2 at a concentration of $1 \mu \mathrm{M})$.

(A)

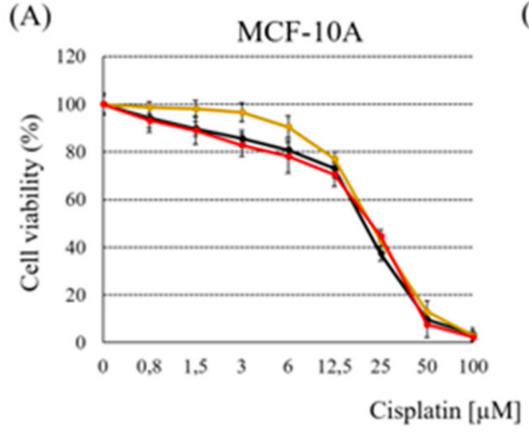

(B)

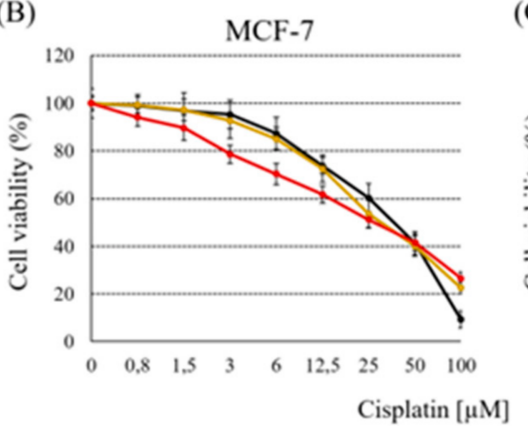

(C)

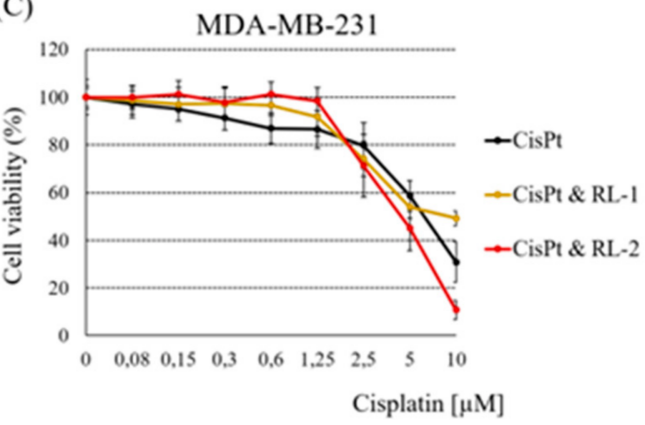

Figure 4. Single (Cisplatin treated cells- black line) and combination drug-response curves for Cisplatin and RL-1 (red line) and Cisplatin and RL-2 (blue line) combinations, based on measurement of cell viability of breast cell lines $48 \mathrm{~h}$ after treatment, analyzed by MTT assay. (A) Data observed for normal MCF-10A cell line, (B) for MCF-7 cells and (C) for MDA-MB-231 cells. All values are expressed as mean $\pm \mathrm{SD}(n=3)$. All variations versus corresponding controls are statistically significant $(p<0.05)$. 
The calculated $\mathrm{IC}_{50}$ values after treatment with Cisplatin alone or in combination with RL-1 and RL-2 are shown in Table 1.

Table 1. Calculated $\mathrm{IC}_{50}$ values (in $\mu \mathrm{M}$ ) $48 \mathrm{~h}$ after treatment of breast cell lines with Cisplatin (concentration range: $0-100 \mu \mathrm{M}$ ) and $1 \mu \mathrm{M}$ from RL-1 or RL-2, estimated through a non-linear regression curve via Origin software and respective \% of change in range of $\mathrm{IC}_{50}$.

\begin{tabular}{cccc}
\hline Cell Lines & CisPt & CisPt + RL-1 Change [\%] & CisPt + RL-2 Change [\%] \\
\hline MCF-10A & 20.96 & $21.95[+4.5 \%]$ & $22.22[+5.7 \%]$ \\
MCF-7 & 32.53 & $30.51[-6.2 \%]$ & $24.58[-24.4 \%]$ \\
MDA-MB-231 & 6.58 & $7.70[+14.5 \%]$ & $4.22[-35.9 \%]$ \\
\hline
\end{tabular}

According to the data presented in Table 1 in the range of $\mathrm{IC}_{50}$ values, the combined treatment with CisPt + RL-2 of both cancer cell lines MCF-7 and MDA-MB-231 exhibited a synergistic effect- $24.4 \%$ and $35.9 \%$, respectively.

The choice of the cytotoxic drug (Cisplatin) for combined treatment experiments was not random. First of all, Cisplatin is a conventionally used antineoplastic agent, which has the ability to crosslink with the purine bases from DNA and interferes with DNA repair mechanisms, causing DNA damage and subsequently inducing apoptosis in cancer cells. Moreover, it has been recently found that Cisplatin treatment induced protective autophagy in many types of cancers, which may contribute to the development of Cisplatin resistance and result in treatment failure [25]. Our results demonstrate that in comparison to cancer cell lines (Figure 4B,C) for normal MCF-10A, the combination with Rhamnolipids (Figure 4A) has no significant effect on Cisplatin cytotoxicity.

This was further confirmed by calculated $\mathrm{IC}_{50}$ values, which were similar: $\mathrm{IC}_{50}$ $(20.96 \mu \mathrm{M})$ for "Cisplatin" versus $\mathrm{IC}_{50}(21.95 \mu \mathrm{M})$ for "Cisplatin and RL-1" versus $\mathrm{IC}_{50}$ $(22.22 \mu \mathrm{M})$ for "Cisplatin and RL-2". This observation we explained with inducted autophagy: formation of membrane vesicles and subsequent degradation and neutralization of drug, respectively absence of toxicity. The images taken from AO staining represent a strong confirmation for this hypothesis. In the case of cancer MCF-7 cells (Figure 4B), both combined treatments could be summarized that after application of biosurfactants, the cytotoxicity of Cisplatin was facilitated. However, better effectiveness was observed after treatment with RL-2 (calculated $\mathrm{IC}_{50}$ values shown in Table 2). Concerning MDAMB-231 cell line: (1) higher sensitivity to anticancer action of Cisplatin was found. The calculated $\mathrm{IC}_{50}$ dose was found to be lower: $6.58 \mu \mathrm{M}$; (2) clear and strong influence on Cisplatin-induced cytotoxicity after combination with Rhamnolipids (Figure 4C) was established. Furthermore, the calculated differences in $\mathrm{IC}_{50}$ dosages were significant after the application of RL-2 and ( 2 times lower): "Cisplatin and RL-1" IC $50(7.70 \mu \mathrm{M})$ versus "Cisplatin and RL-2" $\mathrm{IC}_{50}(4.22 \mu \mathrm{M})$, were demonstrated.

Table 2. $\mathrm{IC}_{50}$ values $[\mu \mathrm{M}]$. Prediction of the effect of combined treatment (Cisplatin and Rhamnolipids) on breast cell lines, based on calculation of the theoretical values of cell viability ("Materials and Methods"). The presented predictions are calculated after combined treatment with concentration: MCF-10A cells: CisPt $(100 \mu \mathrm{M})$ and $1 \mu \mathrm{g} / \mathrm{mL}$ RL-1/ RL-2; MCF-7 cells: CisPt $(100 \mu \mathrm{M})$ and $1 \mu \mathrm{g} / \mathrm{mL}$ RL-1/ RL-2 and MDA-MB-231 cells: CisPt $(10 \mu \mathrm{M})$ and $1 \mu \mathrm{g} / \mathrm{mL}$ RL-1/ RL-2.

\begin{tabular}{ccc}
\hline Cell Lines & CisPt + RL-1 & CisPt + RL-2 \\
\hline MCF-10A & N/A & N/A \\
MCF-7 & Antagonistic & Antagonistic \\
MDA-MB-231 & Antagonistic & Synergistic \\
\hline
\end{tabular}

We tried to predict the type of effect of combined treatment with higher dosages Cisplatin (100 $\mu \mathrm{M}$ Cisplatin for MCF-10A and MCF-7 lines and $10 \mu \mathrm{M}$ for MDA-B-231 cells) and fixed dose $(1 \mu \mathrm{g} / \mathrm{mL}) \mathrm{RLs}$, by calculation of the theoretical values of cell viability (Table 1). In absolute accordance with all data obtained, we predicted that only in the case 
of combined treatment with Cisplatin and RL-2, a synergistic effect could be observed. All other effects were predicted as antagonistic. We proposed that Rhamnolipids act as an autophagy inhibitor with a mechanism related to the LMP of AVOs. The explanation of this fact is that in all other cases, we had predicted an antagonistic effect. In the case of MCF-10A and MCF-7 cells, $\mathrm{IC}_{50}$ values were calculated to be $\sim$ five times higher than $\mathrm{IC}_{50}$ for MDA-MB-231 cells. Therefore, when RL inhibited autophagy for $48 \mathrm{~h}$, the rate of this process is not enough to clear the Cisplatin molecule from the cells, due to its high concentration.

As far as apoptosis and autophagy share the same regulatory mechanisms, in order to determine additional whether the cytotoxic effect of Rhamnolipids is due to the apoptosis, we performed a FACS analysis. The apoptosis in three cell lines was measured by AnnexinV-FITC and propidium iodide staining (Figure 5).
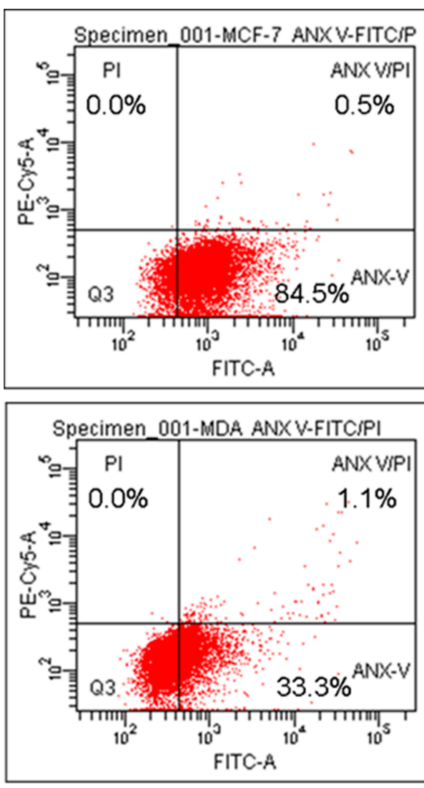

Untreated cells
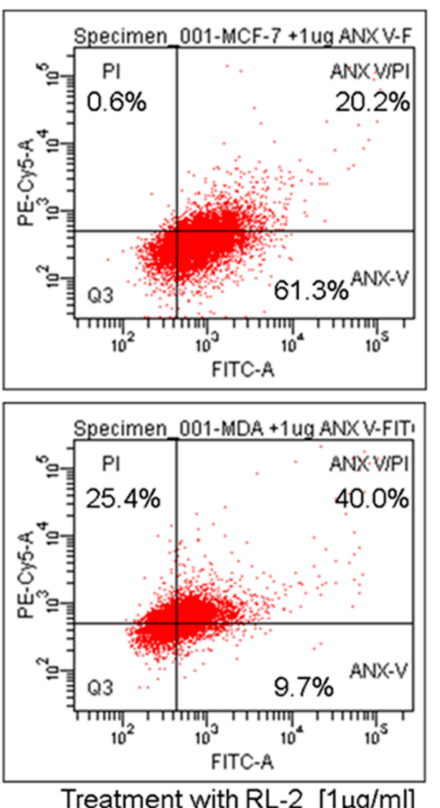
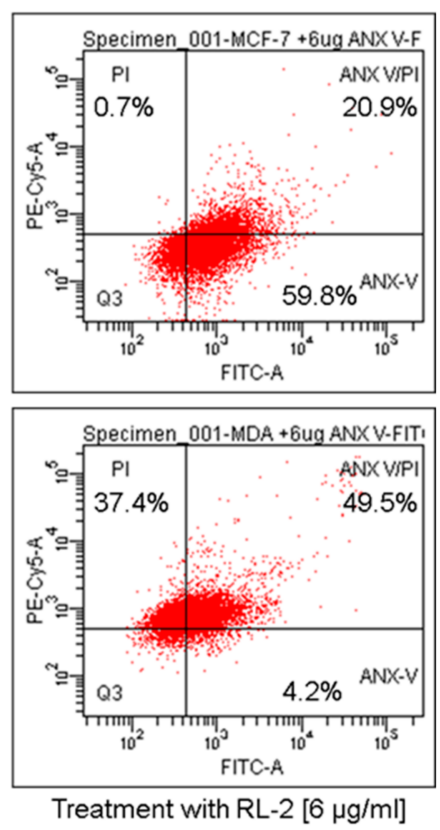

Figure 5. FACS analysis after RL-2 treatment of MCF 7-first row and MDA-MB-231-second row. First colomn corresponds to the untreated cells, second one to the treated cells with RL-2 [1 $\mu \mathrm{g} / \mathrm{mL}]$, third column to RL-2 [6 $\mu \mathrm{g} / \mathrm{mL}]$.

There are two types of apoptosis early apoptosis and late one. During the first phaseearly apoptotic cells remain intact but expose phosphatidylserine on the cell surface. Early apoptosis can become late when the plasma membrane is permeabilized. In Figure 5, the apoptotic assay is presented. Based on our previous data, we chose to treat cells with LR-2 at two different concentrations $1 \mu \mathrm{g} / \mathrm{mL}$ and $6 \mu \mathrm{g} / \mathrm{mL}$. From the figure, we can conclude that MCF7 and MDA-MB-231 react in different ways to the treatment. The triple-negative cell line indicates that after treatment, it develops late apoptosis, progressing to necrosis, with a strong dose-dependent effect, while the low-metastatic line is characterized by an early phase of apoptosis, which is almost independent of the concentration of administrated RL.

The results of the apoptosis assay show a good agreement with the results obtained after the combined treatment of the cell lines with RLs and Cisplatin. Table 2 shows that only in MDA-MB-231 we have a synergistic effect.

As RL are biosustainable, biocompatible and biodegradable surfactants with a lot of practical applications, their physicochemical properties were studied in different types of model biomembranes. Model bilayers with diverse complexity in the presence of RL have been used aiming to receive new insights on lateral membrane organization, morphology and topology. Haba et al. have shown that RLs have increased membrane fluidity and permeability [28]. The molecular interactions of RL with other lipids were studied by 
using giant unilamellar vesicles, homogeneous and heterogeneous ones [10,29]. The latter represented raft-like domains (in liquid-ordered state, Lo) within the membrane in liquiddisordered phase (Ld). Remarkably, in the presence of RLs, it is possible to induce a positive or negative lipid bilayer curvature in dependence on the type of RL molecule. Mono-Rhamnolipids are considered to induce negative membrane curvature due to their cone-like shaped molecule opposite to inverted cone-shaped di-Rhamnolipids, which are able to provoke positive membrane curvature [29]. First, it has been shown that RLs have a low partition in the lipid bilayer with respect to the total molecules in solution. Second, it was suggested that RLs insert in the outer leaflet with a low propensity to flip-flop due to their large polar head [28]. Third, a higher partition coefficient was assessed for the RL of the Ld phase than for the Lo one. Based on these physicochemical properties, it was shown that RLs were able to induce the formation of small quasi-closed buds that remain linked to the original membrane in monophasic PC vesicles and, more spectacularly, to induce Lo domain budding and fission from membranes exhibiting Lo/Ld phase coexistence [28,29]. All these intrinsic properties of the RLs to remodel membrane curvature might be correlated to the endocytic processes activated in cell membranes.

Interestingly, cancer cells were reported to have elevated levels of small, rich of cholesterol and sphingolipid domains in size of 10-200 nm, popular as "lipid rafts" (lipidordered platforms) and they are the place for protein receptors regulating signaling, apoptosis, adhesion, immune responses, redox balance or autophagy. Some antineoplastic agents such as alkylphosphoholins (synthetic antitumor lipids) can express their activity by the reorganization of lipid rafts. Cancer cells contain higher levels of intracellular cholesterol and lipid rafts than their normal, non-tumorigenic counterparts [30-32]. Li et al. reported and paid attention to breast cancer cell lines as MCF-7 and MDA-MB-231, which possess larger cholesterol and GM1 levels (i.e., lipid rafts) compared to their non-tumorigenic counterparts as MCF-10A cells [32].

In our previous work, it was pointed out that RL can induce changes in the membrane morphology of several types of leukemic cells [5]. As mentioned above by phase-contrast imaging, plasma membrane morphological changes and generated vesicular membrane organelles in the breast cancer cells after treatment with Rhamnolipids have been observed. On the other hand, Rahimi et al. [6] reported that treated cancer line MCF-7 with Rhamnolipids have round and shrunken forms, whereas the control cells have cuboidal or polygonal shapes. Based on the data from Figure 1, it is clear that after treatment with RL, the calculated $\left(\mathrm{IC}_{50}\right)$ for malignant cells is lower compared to the normal cell line.

Based on studies on the reorganization of bilayer model membrane in the presence of RL $[29,30]$ and on the availability of recent data on a higher amount of lipid rafts in cancer cell membranes than in their normal ones, non-tumorigenic analogs $[11,31,32]$ and our experimental results, we suggest possible mechanisms in order to explain the observed synergistic effect of RL-2 and Cisplatin on highly metastatic breast cancer cells. It could be suggested that surfactant RLs possessing hydrophobic moiety (long fatty acid chains) predominantly intercalates in the membrane regions in the Ld phase, in particular in the Lo/Ld interface where the lipid packing is perturbed due to the Lo/Ld hydrophobic mismatch. It is known that lipid rafts are thicker than the disordered part of the cell membrane. Thus, the Lo/Ld border represents a region where lower energy is needed to open a space for RLs membrane intercalation. The hydrophilic moiety of RLs is a negatively charged carbohydrate compound, Rhamnose, with surfactant properties, and could reorganize the outer monolayer of Ld regions with a much lower effect on ordered lipid rafts. As we mentioned above, RL-2 possesses an inverted cone shape, and as such, it is possible to fluidize and permeabilize the membrane. Cisplatin itself is also able to fluidize and permeabilize the membrane, in particular in the presence of negatively charged lipids [33]. In turn, the accumulation of inverted cone-shaped lipids with low flip-flop will give layers with different surface asymmetries between the outer (Sout) and inner (Sint) monolayers, as Sout $>$ Sint. Moreover, the lateral diffusion of lipid molecules trapped between ordered domains (in our case, lipid rafts) should be limited according to the 
theoretical model for the "archipelago effect" [34]. Thus, the progressive increase of the surface area of the outer Ld membrane monolayer will form local membrane regions with positive curvature enriched in RL-2 due to the archipelago effect. This fact, in turn, will further increase the permeability of Cisplatin because it was demonstrated that a highly curved membrane $\left(0.2 \mathrm{~nm}^{-1}\right)$ is one to three orders of magnitude more permeable for Cisplatin in comparison to flat or negatively curved ones [35]. It seems that both intrinsic properties of the RL-2 molecule, negative charge and inverted cone shape, further enhance the Cisplatin internalization and effect on the cell cytotoxicity as it is shown as the first possible mechanism in Figure 6a. Shortly, in order to have a synergistic effect (Table 2), it is necessary to have Cisplatin and RL-2, which in turn provide a negative charge and a positive curvature. RL-1 induces negative membrane curvature, which does not act synergistically with Cisplatin to further permeabilize the membrane.
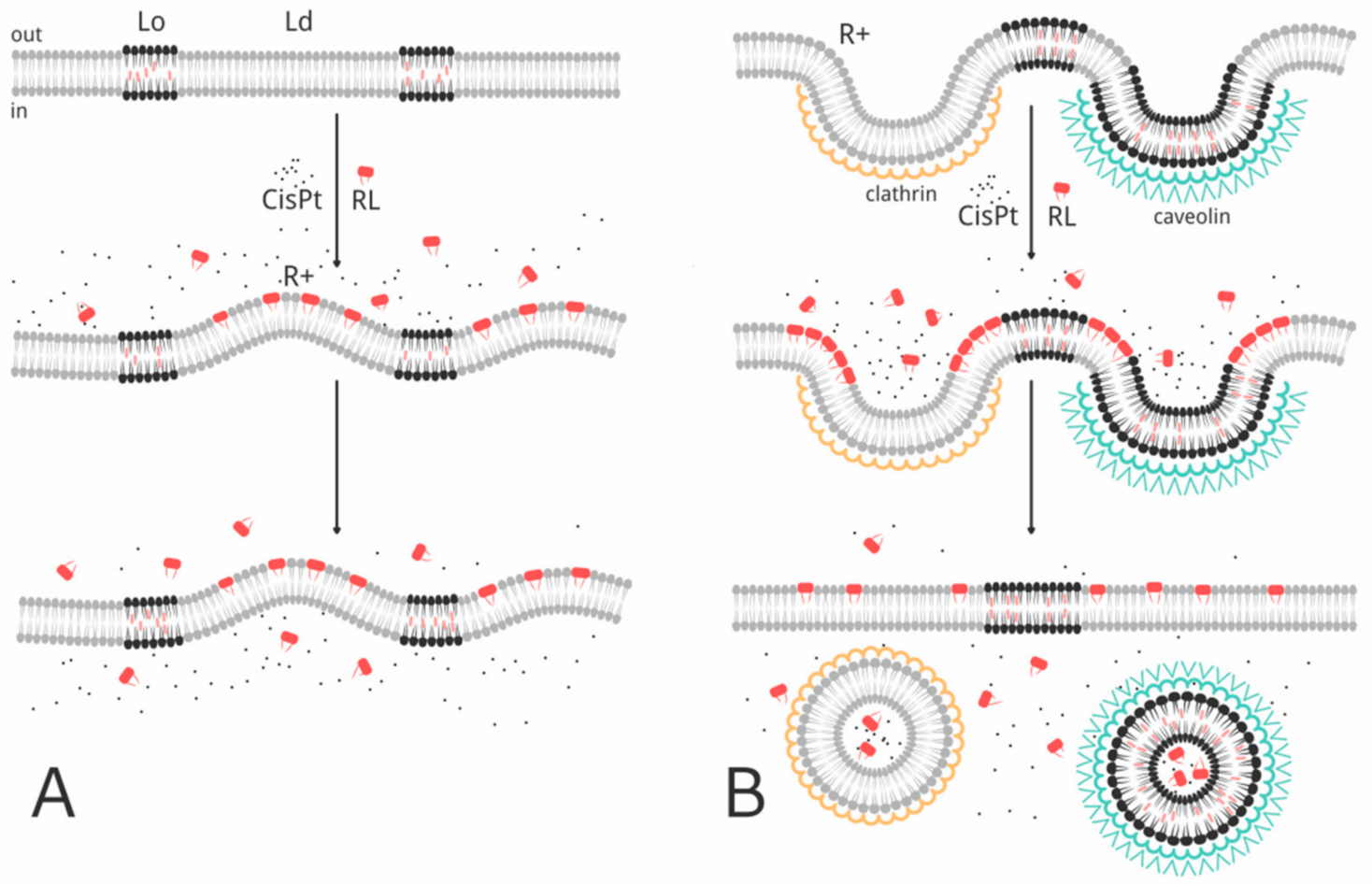

Figure 6. Possible mechanisms of the morphological membrane reorganization and endosomes formation in breast cancer cell induced by the combined action of RL-2 and CisPt. (A) Plasma membrane represents lipid rafts in liquid-ordered phase (Lo) surrounded by lipids in a liquid-disordered state (Ld); RL-2 (red molecules) partition into the membrane and induce positive membrane curvature; synergistic permeabilization of CisPt (black points) induced by inverted cone-shaped RL-2 and the interaction between negative charge of RL-2 and CisPt. (B) Plasma membrane represents clathrin-coated and caveolin pits; RL-2 partition predominantly into the membrane regions characterized with positive membrane curvature such as invagination's necks due to steric similarity; accumulation of permeabilizing molecules such as RL-2 and CisPt favors the fission of invaginated membranes and formation of clathrin and caveolin vesicles containing RL-2 and CisPt.

As we noted above, RL-2 could induce RL-2 enriched Ld budding from the model membrane in the Ld phase state as well as Lo domain budding and fission from membrane exhibiting Lo/Ld phase coexistence. In both cases, the direction of the bud is governed first by the preexisting positive curvature resulting from the vesicle diameter and second by the additionally added positive curvature induced by the accumulation of the inverted cone-shaped molecules. However, in the cells, there are different types of preexisted invaginations, characterized with negative membrane curvature, related or not to rafts, such as caveolin coated pits or clathrin ones. We suggest that this preexisted negative membrane curvature predetermines the direction of the bud, which could be further promoted by RL-2 
and fissioned by synergism of RL-2 and Cisplatin. The most energetically favorable position of RL-2 with its characteristic inverted cone shape is to partition mainly into regions with positive membrane curvatures such as the invagination necks due to steric similarity. As both RL-2 and Cisplatin are able to permeabilize the membrane, it could be hypothesized that the synergistic effect of both molecules is able to form nanopores as it is excepted to be for inverted cone-shaped molecules [36], which, in turn, could lead to fission of the clathrin- and caveolin- dependent invaginations (Figure 6b). Moreover, both the clathrinand caveolin- dependent endocytosis are implicated in autophagy processes $[37,38]$.

Similar morphological transformations of the membranes into large biomimetic liposomes published in our early articles show different membrane invaginations induced by DNA of different lengths [39] as well as Lo domain budding and fission induced by lysophosphatidylcholine as a product of the phospholipase $A_{2}$ hydrolysis [40,41].

The suggested above reorganization of breast cancer cell membrane in the presence of RL-2 and Cisplatin is summarized in Figure 6. The endosomic vesicles and reduced membrane surface of cancer cells were observed by us in the current study as well as in the study of Rahimi, K. et al. [6]. Cisplatin is a small inorganic molecule with a well-expressed cytotoxic effect, but in cancer cells, there are some membrane transporters (multidrug resistance) that take out the drugs from cells. The transport of Cisplatin by endosomes could partly reduce the multidrug resistance. The RL-2 and Cisplatin included in endosomes could provoke the triggering of autophagy or apoptosis in the breast cancer cells.

\section{Conclusions}

To the best of our knowledge, this is the first report demonstrating a Rhamnolipidmediated cytotoxic activity on cancer cells, explained with the LMP and down-regulation of an autophagy process. All data suggest that tested biosurfactants have great potential as natural anticancer agents against breast cancer. Moreover, based on our results, the combined treatment protocols (conventional anticancer drugs and Rhamnolipids) open a wide field for research in view of proposed Rhamnolipid possibilities for the inhibition of autophagy, respectively partly overcoming drug resistance.

Author Contributions: B.N., S.S. and G.A. conceived the idea for the manuscript and produced the first draft. G.A., S.S. and I.I. conducted the experiments. N.C., L.N., I.S. and L.K. participated in cultivation of the strain for RL production and purification process. P.L. was involved in image preparation and improvements. I.T., B.N., L.K. and G.S. were involved in the critical review of the drafts and final version. All authors read and approved the final version of the manuscript.

Funding: This research was funded by the Bulgarian National Research Fund (grant DN11/2 (2017) to B.N., and grant KP-06-COST/10 (2020) B.N.).

Institutional Review Board Statement: Not applicable.

Informed Consent Statement: Not applicable.

Acknowledgments: To COST Action COMULIS CA171121.

Conflicts of Interest: The authors declare no conflict of interest.

\section{References}

1. Ron, E.Z.; Rosenberg, E. Natural roles of biosurfactants. Environ. Microbiol. 2001, 3, 229-236. [CrossRef] [PubMed]

2. Sekhon Randhawa, K.K.; Rahman, P.K. Rhamnolipid biosurfactants-past, present, and future scenario of global market. Front. Microbiol. 2014, 5, 454. [CrossRef] [PubMed]

3. Marchant, R.; Banat, I.M. Microbial biosurfactants: Challenges and opportunities for future exploitation. Trends Biotechnol. 2012, 30, 558-565. [CrossRef] [PubMed]

4. Chen, J.; Wu, Q.; Hua, Y.; Chen, J.; Zhang, H.; Wang, H. Potential applications of biosurfactant rhamnolipids in agriculture and biomedicine. Appl. Microbiol. Biotechnol. 2017, 101, 8309-8319. [CrossRef] [PubMed]

5. Christova, N.; Tuleva, B.; Kril, A.; Georgieva, M.; Konstantinov, S.; Terziyski, I.; Nikolova, B.; Stoineva, I. Chemical structure and in vitro antitumor activity of rhamnolipids from Pseudomonas aeruginosa BN10. Appl. Biochem. Biotechnol. 2013, 170, 676-689. [CrossRef] 
6. Rahimi, K.; Lotfabat, T.B.; Jabeen, F.; Ganji, S.M. Cytotoxic effect of mono-and di-rhamnolipids from Pseudomons aeruginosa MR01 on MCF-7 human breast cancer cells. Colloids Surf. B Biointerfaces 2019, 181, 943-952. [CrossRef]

7. Thanomsub, B.; Pumeechockchai, W.; Limtrakul, A.; Arunrattiyakorn, P.; Petchleelaha, W.; Nitoda, T.; Kanzaki, H. Chemical structures and biological activities of rhamnolipids produced by Pseudomonas aeruginosa B189 isolated from milk factory waste. Bioresour. Technol. 2006, 97, 2457-2461. [CrossRef]

8. Zhao, J.; Wu, Y.; Alfred, A.T.; Xin, X.; Yang, S. Chemical structure and biological activities of Rhamnolipid biosurfactants produced by Pseudomonas aeruginosa M14808. J. Chem. Pharm. Res. 2013, 5, 177-182.

9. Sanches, M.; Aranda, J.A.; Teruel, J.A.; Espuny, M.J.; Marques, A.; Manresa, A.; Ortiz, A. Permeabilization of biological and artificial membranes by a bacterial dirhamnolipid produced by pseudomonas aeruginosa. J. Colloid Interface Sci. 2010, 341, 240-247. [CrossRef]

10. Come, B.; Donato, M.; Potenza, L.F.; Mariani, P.; Itri, R.; Spinozzi, F. The intriguing role of rhamnolipids on plasma membrane remodelling: From lipid rafts to membrane budding. J. Colloid Interface Sci. 2021, 582, 669-677. [CrossRef]

11. Gudiña, E.J.; Rangarajan, V.; Sen, R.; Rodrigues, L.R. Potential therapeutic applications of biosurfactants. Trends Pharamacol. Sci. 2013, 34, 667-675. [CrossRef]

12. Chena, S.; Rehman, S.K.; Zhang, W.; Wen, A.; Yao, L.; Zhang, J. Autophagy is a therapeutic target in anticancer drug resistance. BBA Rev. Cancer 2010, 1806, 220-229. [CrossRef]

13. Yang, Z.; Klionsky, D.J. An overview of the molecular mechanism of autophagy. Curr. Top. Microbiol. Immunol. 2009, 335, 1-32. [PubMed]

14. Klionsky, D.J.; Emr, S.D. Autophagy as a regulated pathway of cellular degradation. Science 2000, 290, 1717-1721. [CrossRef] [PubMed]

15. Janku, F.; McConkey, D.J.; Hong, D.S.; Kurzrock, R. Autophagy as a target for anticancer therapy. Nat. Rev. Clin. Oncol. 2011, 8, 528-539. [CrossRef] [PubMed]

16. Tuleva, B.; Hristova, N.; Cohen, R.; Stoev, G.; Stoineva, I. Production and structural elucidation of trehalose tetraesters (biosurfactants) from a novel alkanothrophic rhodococcus wratislaviensis strain. J. Appl. Microbiol. 2008, 104, 1703-1710. [CrossRef] [PubMed]

17. Mosmann, T. Rapid colorimetric assay for cellular growth and survival: Application to proliferation and cytotoxicity assays. J. Immunol. Method. 1983, 65, 55-63. [CrossRef]

18. Georgieva, M.; Konstantinov, S.; Topashka-Ancheva, M.; Berger, M.R. Combination effects of alkylphosphocholines and gemcitabine in malignant and normal hematopoietic cells. Cancer Lett. 2002, 182, 163-174. [CrossRef]

19. Akiyode, O.; George, D.; Getti, G.; Boateng, J. Systematic comparison of the functional physico-chemical characteristics and biocidal activity of microbial derived biosurfactants on blood-derived and breast cancer cells. Colloid Interface Sci. 2016, 479, 221-233. [CrossRef]

20. Jiang, L.; Shen, C.; Long, X.; Zhang, G.; Meng, Q. Rhamnolipids elicit the same cytotoxic sensitivity between cancer cell and normal cell by reducing surface tension of culture medium. Appl. Microbiol. Biotechnol. 2014, 98, 10187-10196. [CrossRef]

21. Galluzzi, L.; Pietrocola, F.; Bravo-San Pedro, J.M.; Amaravadi, R.K.; Baehrecke, E.H.; Cecconi, F.; Codogno, P.; Debnath, J.; Gewirtz, D.A.; Karantza, V.; et al. Autophagy in malignant transformation and cancer progression. EMBO J. 2015, 34, 856-880. [CrossRef]

22. Kondo, Y.; Kanzawa, T.; Sawaya, R.; Kondo, S. The role of autophagy in cancer development and response to therapy. Nat. Rev. Cancer 2005, 5, 726-734. [CrossRef]

23. Thomé, M.P.; Filippi-Chiela, E.C.; Villodre, E.S.; Migliavaca, C.B.; Onzi, G.R.; Felipe, K.B.; Lenz, G. Ratiometric analysis of Acridine Orange staining in the study of acidic organelles and autophagy. J. Cell Sci. 2016, 129, 4622-4632. [CrossRef]

24. Chiu, C.F.; Chin, H.K.; Huang, W.J.; Bai, L.Y.; Huang, H.Y.; Weng, J.R. Induction of apoptosis and autophagy in breast cancer cells by a novel hdac8 inhibitor. Biomolecule 2019, 9, 824. [CrossRef] [PubMed]

25. Lin, J.F.; Lin, Y.C.; Tsai, T.F.; Chen, H.E.; Chou, K.Y.; Hwang, T.I. Cisplatin induces protective autophagy through activation of BECN1 in human bladder cancer cells. Drug. Des. Dev. Ther. 2017, 11, 1517-1533. [CrossRef] [PubMed]

26. Wang, F.; Gómez-Sintes, R.; Boya, P. Lysosomal membrane permeabilization and cell death. Traffic 2018, 19, 918-931. [CrossRef] [PubMed]

27. Serrano-Puebla, A.; Boya, P. Lysosomal membrane permeabilization as a cell death mechanism in cancer cells. Biochem. Soc. Trans. 2018, 46, 207-215. [CrossRef]

28. Haba, E.; Pinazo, D.; Pons, R.; Perez, L.; Manresse, D. Complex rhamnolipid mixture characterization and influence on DPPC bilayers organization. Biochim. Biophys. Acta Biomebranes 2014, 1838, 776-783. [CrossRef] [PubMed]

29. Herzog, M.; Tiso, T.; Blank, L.M.; Winter, R. Interaction of rhamnolipids with model biomembranes of varying complexity. Biochim. Biophys. Acta Biomebranes 2020, 18862, 183431. [CrossRef]

30. Mollinedo, F.; Gajate, G. Lipid rafts as signaling hubs in cancer cell survival/death and invasion: Implications in tumor progression and therapy. J. Lip. Res. 2020, 61, 611-628. [CrossRef]

31. Kaleagsioglu, F.; Ali, D.M.; Berger, M.R. Multiple facets of autophagy and the emerging role of alkylphosphocholines as autophagy modulators. Front. Pharmacol. 2020, 11, 547. [CrossRef]

32. Li, Y.C.; Park, M.J.; Ye, S.K.; Kim, C.W.; Kim, Y.N. Elevated levels of cholesterol-rich lipid rafts in cancer cells are correlated with apoptosis sensitivity induced by cholesterol-depleting agents. Am. J. Pathol. 2006, 168, 1107-1118. [CrossRef] 
33. Martinho, N.; Santos, T.C.; Florindo, H.F.; Silva, L.C. Cisplatin-membrane interactions and their influence on platinum complexes activity and toxicity. Front. Physiol. 2019, 9, 1898. [CrossRef]

34. Saxton, M.G. Lateral diffusion in an archipelago. Dependence on tracer size. Biophys. J. 1993, 64, 1053-1062. [CrossRef]

35. Yesylevskyy, S.; Rivel, T.; Ramseyer, C. Curvature increases permeability of the plasma membrane for ions, water and the anti-cancer drugs cisplatin and gemcitabine. Sci. Rep. 2019, 9, 17214. [CrossRef] [PubMed]

36. Zhelev, D.V. Material property characteristics for lipid bilayers containing lysolipid. Biophys. J. 1998, 75, 321-330. [CrossRef]

37. Birgisdottir, Å.B.; Johansen, T. Autophagy and endocytosis-Interconnections and interdependencies. J. Cell Sci. 2020, 133 , jcs228114. [CrossRef]

38. Zhang, X.; Ramírez, C.M.; Aryal, B.; Madrigal-Matute, J.; Liu, X.; Diaz, A.; Torrecilla-Parra, M.; Suárez, Y.; Cuervo, A.M.; Sessa, W.C.; et al. Cav-1 (Caveolin-1) deficiency increases autophagy in the endothelium and attenuates vascular inflammation and atherosclerosis. Arterioscler. Thromb. Vasc. Biol. 2020, 40, 1510-1522. [CrossRef]

39. Angelova, M.; Tsoneva, I. Interactions of DNA with giant liposomes. Chem. Phys. Lipids 1999, 101, 123-127. [CrossRef]

40. Staneva, G.; Angelova, M.I.; Koumanov, K. Phospholipase A2 promotes raft budding and fission from giant liposomes. Chem. Phys. Lipids 2004, 129, 53-62. [CrossRef]

41. Georgieva, R.; Mircheva, K.; Vitkova, V.; Balashev, K.; Ivanova, T.; Tessier, S.; Koumanov, K.; Nuss, P.; Momchilova, A.; Staneva, G. Phospholipase A2-induced remodeling processes on liquid-ordered/liquid-disordered membranes containing docosahexaenoic or oleic acid: A comparison study. Langmuir 2016, 32, 1756-1770. [CrossRef] [PubMed] 